\title{
EXPERIMENTAL INVESTIGATION OF FLOW INSTABILITIES AND ROTATING STALL IN A HIGH-ENERGY CENTRIFUGAL PUMP STAGE
}

\author{
Stefan Berten \\ Laboratory for Hydraulic \\ Machines, EPFL \\ Lausanne, Switzerland \\ Email:stefan.berten@epfl.ch \\ Maher Kayal \\ Institute of Electrical \\ Engineering, EPFL \\ Lausanne, Switzerland \\ Email: maher.kayal@epfl.ch
}

\author{
Philippe Dupont \\ Sulzer Pumps Ltd. \\ Winterthur, Switzerland \\ Email: philippe.dupont@sulzer.com
}

\author{
Francois Avellan \\ Laboratory for Hydraulic \\ Machines, EPFL \\ Lausanne, Switzerland \\ Email: francois.avellan@epfl.ch
}

\author{
Laurent Fabre \\ Institute of Electrical \\ Engineering, EPFL \\ Lausanne, Switzerland \\ Email: laurent.fabre@epfl.ch \\ Mohamed Farhat \\ Laboratory for Hydraulic \\ Machines, EPFL \\ Lausanne, Switzerland \\ Email: mohamed.farhat@epfl.ch
}

\begin{abstract}
In centrifugal pumps, the interaction between the rotating impeller and the stationary diffuser generates specific pressure fluctuation patterns. When the pump is operated at off design conditions, these pressure fluctuations increase. The resulting rise of mechanical vibration levels may negatively affect the operational performance and the life span of mechanical components. This paper presents detailed pressure fluctuation measurements performed in a high speed centrifugal pump stage at full scale at various operating conditions. The impeller and stationary part (diffuser, exit chamber) of the pump stage have been equipped with piezo-resistive miniature pressure sensors. The measured data in the impeller have been acquired using a newly developed onboard data acquisition system, designed for rotational speeds up to $6000 \mathrm{rpm}$. The measurements have been performed synchronously in the rotating and stationary domains. The analysis of pressure fluctuations at the impeller blade trailing edge, which had significantly larger amplitudes as the pressure fluctuations in the stationary domain, allowed the detection and exploration of stalled channels in the vaned diffuser. This stall may be stationary or rotating with different rotational speeds and number of stalled channels, depending on the relative flow rate and the rotational speed of the pump. The stall yields
\end{abstract}

pressure fluctuations at frequencies which are multiples of the rotational speed of the impeller and generates additional sources of mechanical excitation.

\section{NOMENCLATURE}

$c_{\tilde{p}} \quad$ Fluctuating pressure coefficient, $\frac{2 \cdot \tilde{p}}{\rho \cdot u_{2}^{2}},[-]$

$\mathrm{D}_{2} \quad$ Impeller outer diameter, [m]

$f \quad$ Frequency, $[\mathrm{Hz}]$

$f_{B} \quad$ Impeller blade passing frequency, $[\mathrm{Hz}]$

$f_{V} \quad$ Diffuser vane passing frequency, $[\mathrm{Hz}]$

$f_{N} \quad$ Rotational frequency $f_{N}=n / 60,[\mathrm{~Hz}]$

$n \quad$ Rotational speed, [rpm]

$n_{q} \quad$ Specific speed, $n_{q}=n \cdot \frac{Q^{0.5}}{H^{0.75}}[-]$

$p$ Pressure, $[\mathrm{Pa}]$

$\bar{p} \quad$ Mean pressure, $[\mathrm{Pa}]$

$\tilde{p} \quad$ Fluctuating pressure, $\tilde{p}=p-\bar{p},[\mathrm{~Pa}]$

$q * \quad$ Relative flow rate, $q *=Q / Q_{B E P}[-]$

$t$ Time, [s]

$u_{2}$ Impeller circumferential velocity $u_{2}=\frac{\pi \cdot D_{2} \cdot n}{60}[\mathrm{~m} / \mathrm{s}]$

$z_{B} \quad$ Number of impeller blades, [-]

$z_{V} \quad$ Number of diffuser channels, [-] 
$\rho \quad$ Fluid density, $\left[\mathrm{kg} / \mathrm{m}^{3}\right]$

$\theta \quad$ Angular coordinate, $[\mathrm{rad}]$

\section{INTRODUCTION}

At flow rates lower than the design flow rate, flow separations can occur in the rotating as well as in stationary components of centrifugal pumps. These flow separations affect negatively the operational behavior of these machines causing reduced efficiency, higher pressure pulsations and increased vibration levels. A variety of numerical and experimental studies on flow separation and unsteady phenomena in centrifugal compressors have been published (Frigne and Van Den Braembussche [1], Haupt et al. [2] Filipenco et al. [3], Deniz et al. [4]), while the number of research publications regarding centrifugal pumps is relatively limited. Hergt and Benner [5] analyzed different diffusers in a free surface test stand at very low rotational speeds. At different flow rates and different hydraulic designs (diffuser vane number and vane inlet angle), different rotating and non-rotating stall patterns have been visually observed. From these observations they derived design recommendations for multistage pump diffusers based on the stagger angle and the relative camber length of the diffuser blade. Arndt et al. [6] performed pressure fluctuation measurements in the impeller and different diffusers at varying radial gaps between the impeller and the diffuser. He observed sidebands in the impeller pressure fluctuation spectra generated by a modulation between the vane passing frequency and the rotation frequency due to an uneven circumferential pressure distribution. Guo and Maruta [7] performed pressure fluctuation measurements in a centrifugal pump impeller and they found at high flow rates sidebands in the pressure fluctuation spectra. These sidebands were also a result of a modulation of the vane passing frequency with an uneven circumferential pressure distribution. Eisele et al. [8] used LDV and PTV techniques for a detailed flow analysis in a centrifugal pump diffuser at different operating points of the pump. At part-load flow rate they observed recirculation from the diffuser back into the impeller. Sano et al. [9] used CFD for the numerical simulation of the flow in a diffuser connected to an impeller using a moving grid method. The calculations were made "quasi" 2D, the mesh in the ground view was only one element high. Resulting from the simulation, diffuser rotating stall rotating with $10 \%$ of the rotational speed has been found. The simulated flow pattern was in accordance with the measurements of Hergt and Benner [5], Sinha et al. [10] and Wang and Tsukamoto [11]. While in the case studied by Sinha the gap between diffuser and impeller vanes was relatively large (20\% of impeller radius), in Wang's analysis the gap was only $3 \%$ of the impeller outer radius. They found diffuser stall rotating with $1.2 \%$ of the impeller rotational speed.

In the present article we present pressure fluctuation measurements, performed synchronously in the impeller and in the diffuser of a high-energy centrifugal pump at different flow rates and rotational speeds. During these measurements stationary and rotating stall in the diffuser has been observed. The newly designed onboard data acquisition system is described and the measurement results are presented in the time and frequency domain.

\section{EXPERIMENTAL SETUP Test Pump and Test Loop}

The experiments have been performed with a pump model representing the last stage of a high head multistage centrifugal pump with a specific speed of $n_{q}=21.5$. The pump model consists of an inlet casing(1), the 7 blades impeller(2), the vaned diffuser(3), having 12 vanes and an annular ring guiding the flow to the discharge pipe (4). The relative gap between the impeller trailing edge and the diffuser leading edge was $5.8 \%$. Between the inlet casing and the impeller, a return section has been placed in order to simulate the inlet conditions of the last stage of a multistage pump (5). The pump has been installed in a closed test

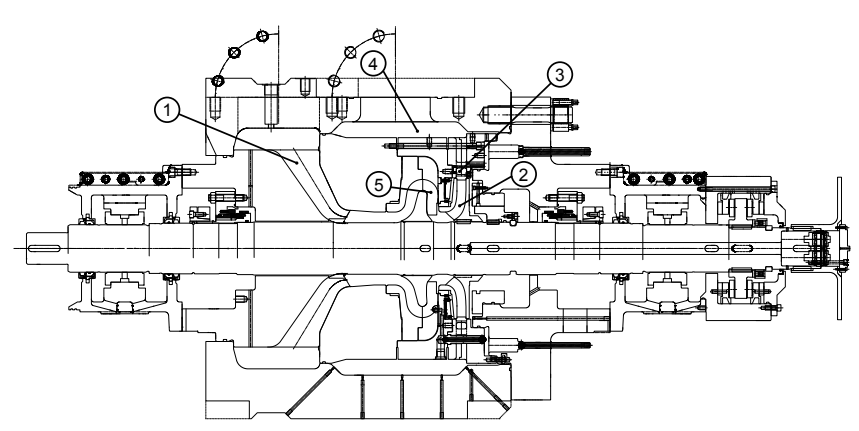

Figure 1. SINGLE STAGE MODEL PUMP

loop, equipped with a high pressure reservoir in order to provide the necessary suction pressure for a cavitation free operation of the test pump at all tested flow rates of interest. The pump has been driven by a variable speed drive which allowed rotational speeds up to $5800 \mathrm{rpm}$.

\section{Instrumentation in rotating and stationary components}

Pressure sensors have been installed in the impeller and the diffuser. The impeller has been equipped with 17 pressure sensors, distributed in the impeller eye, the impeller channels, in the hub and shroud side wall at the impeller outlet, and in the impeller vane close to the trailing edge. The diffuser has been equipped with 31 pressure sensors circumferentially distributed in the diffuser channel throats and outlet sections (see Fig.2). All pressure sensors were flush mounted. The pressure sen- 

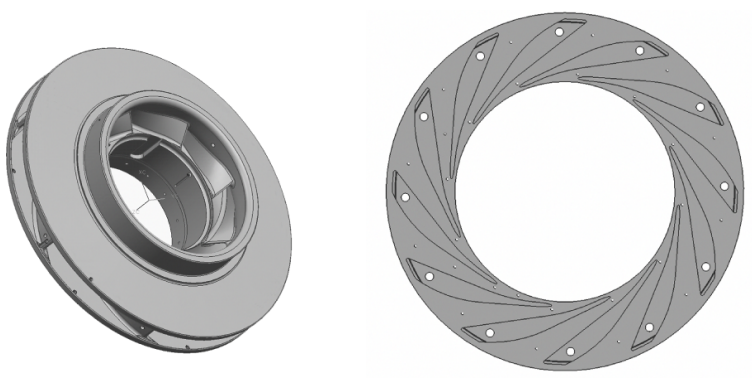

Figure 2. INSTRUMENTED IMPELLER AND DIFFUSER

sors were piezo-resistive miniature pressure transducers made by UNISENSOR AG allowing the measurement of both, unsteady and static pressure applied to the sensor surface. They contain sensitive elements arranged in a Wheatstone bridge under a silicone membrane having a diameter of $3 \mathrm{~mm}$. The pressure sensors have been integrated in specific housings which have been glued in the prepared positions in the impeller and the diffuser. After the sensor embedding, the cables were passed through grooves to a central bore in the shaft leading to the the non-drive end of the pump, where they are connected to two rugged circular connectors having a total of 96 contacts. The cable grooves have been covered with a ceramic composite in order to protect the sensors back face and cables from ingress of moisture. Before the final
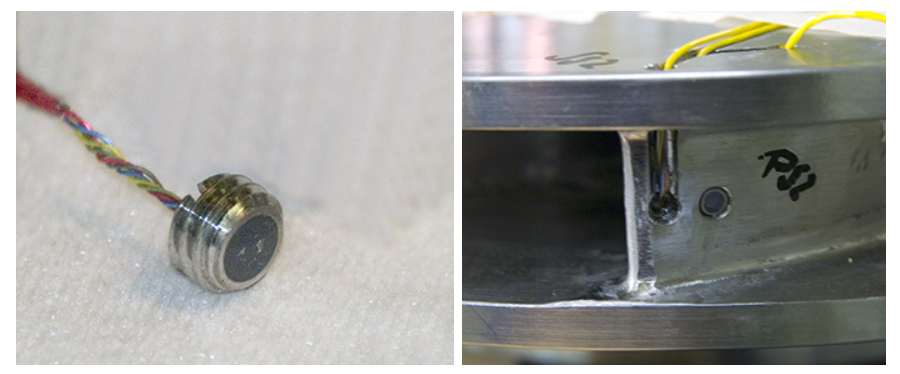

Figure 3. PRESSURE SENSOR IMPLEMENTED IN IMPELLER BLADE

installation in the impeller and the diffuser, the pressure sensors have been statically calibrated in a high pressure test stand with pressures up to 100 bars. The maximum error of the static pressure was estimated to be less than $1 \%$ of the measurement range of the pressure sensor. For the sensors mounted in the impeller blade surface at the oulet, the influence of the rotation has been analytically evaluated and found to be less than $0.2 \%$ and has been therefore neglected during the measurements. The static calibration has been repeated on site in order to verify the influence of the sensor mounting on its behaviour. The obtained calibration curves showed good agreement with the original calibration. The used pressure sensors usually show a drift of their offsets. This drift has been corrected prior to each test by reestimating the offset of each sensor with the help of a reference pressure sensor. The dynamic response of this sensor type has been validated on a sensor identical in construction [12]. The frequency response to different excitations showed an excellent coherence between 0 and $25 \mathrm{kHz}$.

The rotational speed of the pump has been measured using a proximity probe mounted in the bearing housing at the nondrive end of the pump. The shaft has been equipped with one bore, which generated at each passage of the proximity sensor a peak in the voltage output. This keyphasor signal also allows the identification of each revolution of the impeller, which is used for the calculation of phase averages and further analysis of the measured pressure fluctuation data.

\section{Data Acquisition}

The stationary domain pressure fluctuation data and the reference signal for the shaft angular position have been acquired using a VXI HP1432A data acquisition system. With this system it was possible to acquire dynamic pressure data with sampling frequencies up to $51.2 \mathrm{kHz}$ and a resolution of $16 \mathrm{bit}$. The memory is sufficient to store 1'048'576 samples per channel. Prior to the acquisition the pressure sensor signals have been amplified using in-house developed preamplifiers, the anti aliasing filters were integrated in the data acquisition system.

Based on available experiences at the EPFL-LMH ( [13], [14]), a new onboard conditioning and data acquisition system has been developed to allow pressure fluctuation measurements at high rotational speeds. This demand required a miniaturization and optimization of mechanical as well as electronical components of the onboard data acquisition system. The new onboard system consists of 32 measurement channels mounted on 4 circular multilayer boards. The positioning of the electronical components has been optimized to account for the high mechanical load due to the centrifugal forces at the intended rotational speeds. The onboard data acquisition system is capable to acquire voltage data with sampling frequencies up to $100 \mathrm{kHz}$. The resolution of the digitalization is 12 bit. The memory of the onboard system allows the storage of 450'000 samples for each of the 32 channels. The conditioning electronics, consisting of preamplifiers, anti aliasing filters and a second amplifier are also integrated on the acquisition boards. The amplification factors can be selected between 10 and 800. Software controllable offsets before the preamplifiers and after the anti aliasing filters allow the centering of the signal for an optimal use of the digitalization range. The control and communication is performed with a FPGA card using the USB2 protocol, ensuring a reliable high speed communication between the host computer and the onboard data acquisition system. Power supply, communication with the computer controlling the measurements and external triggering has been realized using a high speed slip ring. The onboard data acqui- 

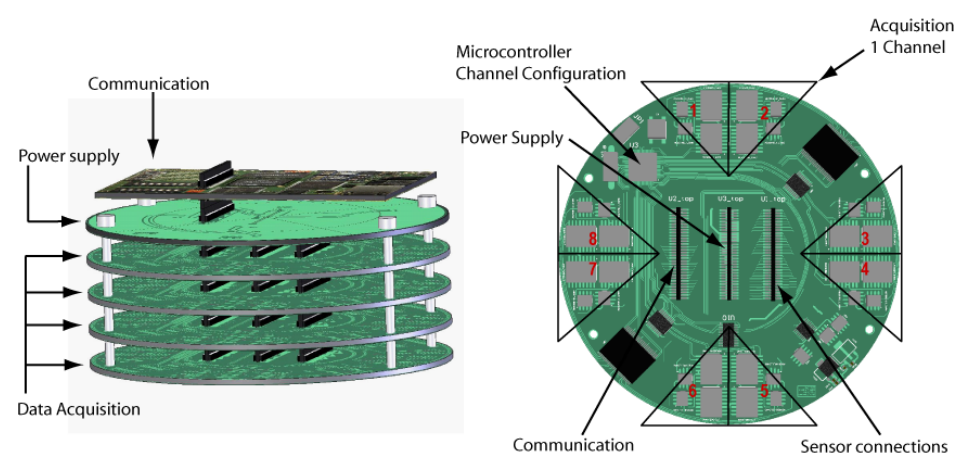

Figure 4. DATA ACQUISITION BOARDS

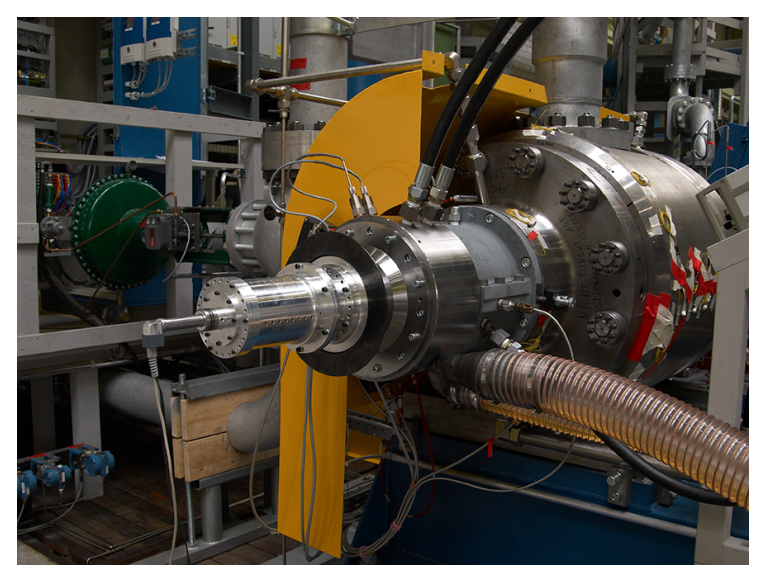

Figure 5. ONBOARD DATA ACQUISITION SYSTEM INSTALLED AT PUMP SHAFT

sition system has been mounted in to a cylindrical casing made of aluminum in order to reduce the systems mass, which was 6 $\mathrm{kg}$. After precision balancing of the whole data acquisition system, it has been fixed overhung on the non-drive end of the pump shaft. The influence of the additional mass on the shaft end on the rotodynamic behavior of the pump has been validated during the design process. During the experiments, a protection cover has been installed around the rotating data acquisition system. The data in the stationary and in the rotating domain have been acquired synchronously by simultaneous triggering of both data acquisition systems using the keyphasor signal.

\section{MEASUREMENT RESULTS Rotating domain data}

Figure 6 shows the phase-averaged, non dimensional fluctuating pressure measured close to the impeller blade trailing edge in the pressure surface with an impeller rotational speed of $1500 \mathrm{rpm}$. The dotted lines represent the limits given by the standard deviation for each circumferential position. At the best efficiency flow rate $\left(\mathrm{q}^{*}=1\right)$, a periodic pattern with 12 maxima
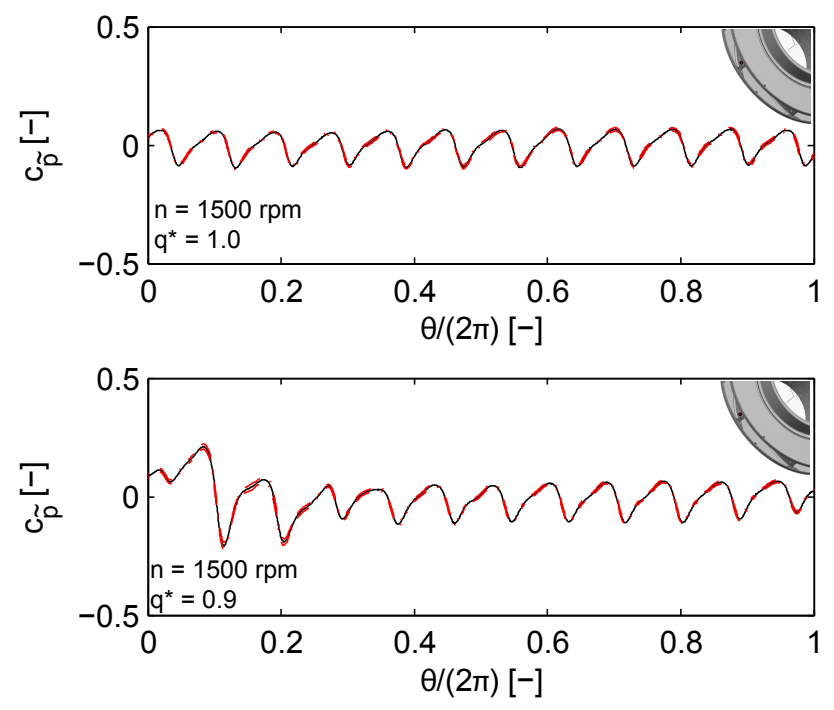

Figure 6. PHASE AVERAGED PRESSURE FLUCTUATIONS AT IMPELLER TRAILING EDGE FOR DIFFERENT RELATIVE FLOW RATES

and 12 minima can be observed. The minima occur, when the impeller blade sensor passes the low pressure zone of the suction surface of the diffuser vanes. The maxima are reached during the passage between 2 diffuser vanes. Fig.7 illustrates the relative impeller blade position for a pressure minimum (left) and a pressure maximum (right). After reducing the flow rate to $90 \%$ of the

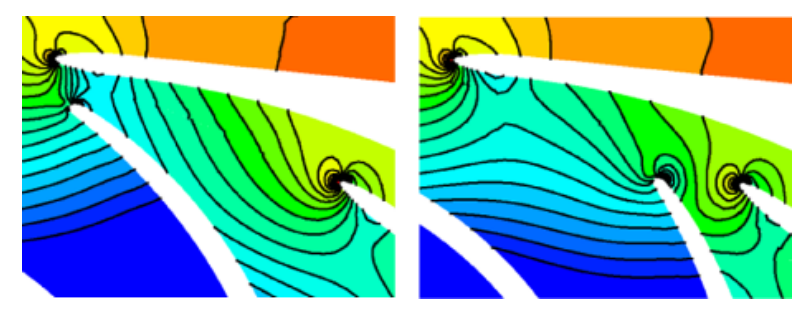

Figure 7. BLADE POSITON CORRESPONDING TO MINIMUM PRESSURE (LEFT) AND MAXIMUM PRESSURE (RIGHT)

best efficiency flow rate, a singular high pressure peak is superposed to the periodic pattern due to the diffuser vane passage. We attribute this local increase of the pressure to a flow separation in one of the diffuser channels. This separation yields an increased diffuser throat pressure due to the reduced flow velocity in the separated diffuser channel. The pressure peak is followed by a pressure minimum, which is caused by the local increase of the flow velocity for the diffuser vane suction surface following the separated diffuser channel in the rotational direction of the impeller. In order to verify this observation, the instantaneous pressure values at different circumferential positions of the impeller 
are represented in Fig. 8 as a waterfall plot, which will help to identify periodic and non periodic variations in the circumferential pressure distribution. At the best effiency flow rate, the non disturbed pressure distribution with the 12 minima and 12 maxima can be identified. At $\mathrm{q}^{*}=0.9$, the stationary separation of one diffuser channel is visible as a high pressure peak. At a flow rate reduced to $\mathrm{q}^{*}=0.8$, this high pressure peak starts to rotate with a rotational speed of less than $0.7 \%$ of the impeller rotational speed. The peak is steadily propagating in the same direction as the impeller rotation direction. At further reduced relative flow rates, the number of pressure peaks, which are propagating in the impeller direction, increases until no real organization of the pressure peaks can be identified at flow rates lower than $50 \%$ of the best efficiency flow rate. Figure 9 presents a waterfall plot of the pressure fluctuation amplitudes measured in the impeller blade pressure surface close to the trailing edge. The spectra for each flow rate have been estimated as the average of 9 single spectra calculated using a Fast Fourier Transform. The resolution of these spectra is $1 \mathrm{~Hz}$, a window function has been applied in order to avoid spectral leakage. Each single amplitude spectrum covered several impeller rotations. At the best efficiency flow rate, the most significant peaks are found at the diffuser vane passage frequency and its harmonics. A small amplitude peak at the rotational frequency $f_{N}$ and very small amplitude sidebands are visible at $f=f_{V} \pm f_{N}$. At part load, when the separation occurs, additional spectral lines at multiples of the rotational speed appear, while the diffuser vane passing frequency amplitude is increasing. The amplitude distribution of these additional lines depend on the number of stalled diffuser channels. These additional spectral lines can be interpreted as the result of a modulation of the vane passing frequency with the pressure peaks in the stalled diffuser channels. When the stationary stall appears at $\mathrm{q}^{*}=0.9$, the first peak appears at the rotational frequency, followed by further peaks at multiples of the rotational frequency, which are the harmonics of this first peak. The frequencies of the side bands are not affected by the rotation of the stall, because the rotational frequency of the stall is significantly smaller than the resolution of the spectra. At lower part load $\left(\mathrm{q}^{*}=0.6\right)$, when the number of stalled cells is increased to 3 , a local maximum in the amplitude spectrum can be observed at $f=f_{V} \pm 3 \cdot f_{N}$, indicating a strong modulation between the vane passing frequency and the frequency of the stalled cells.
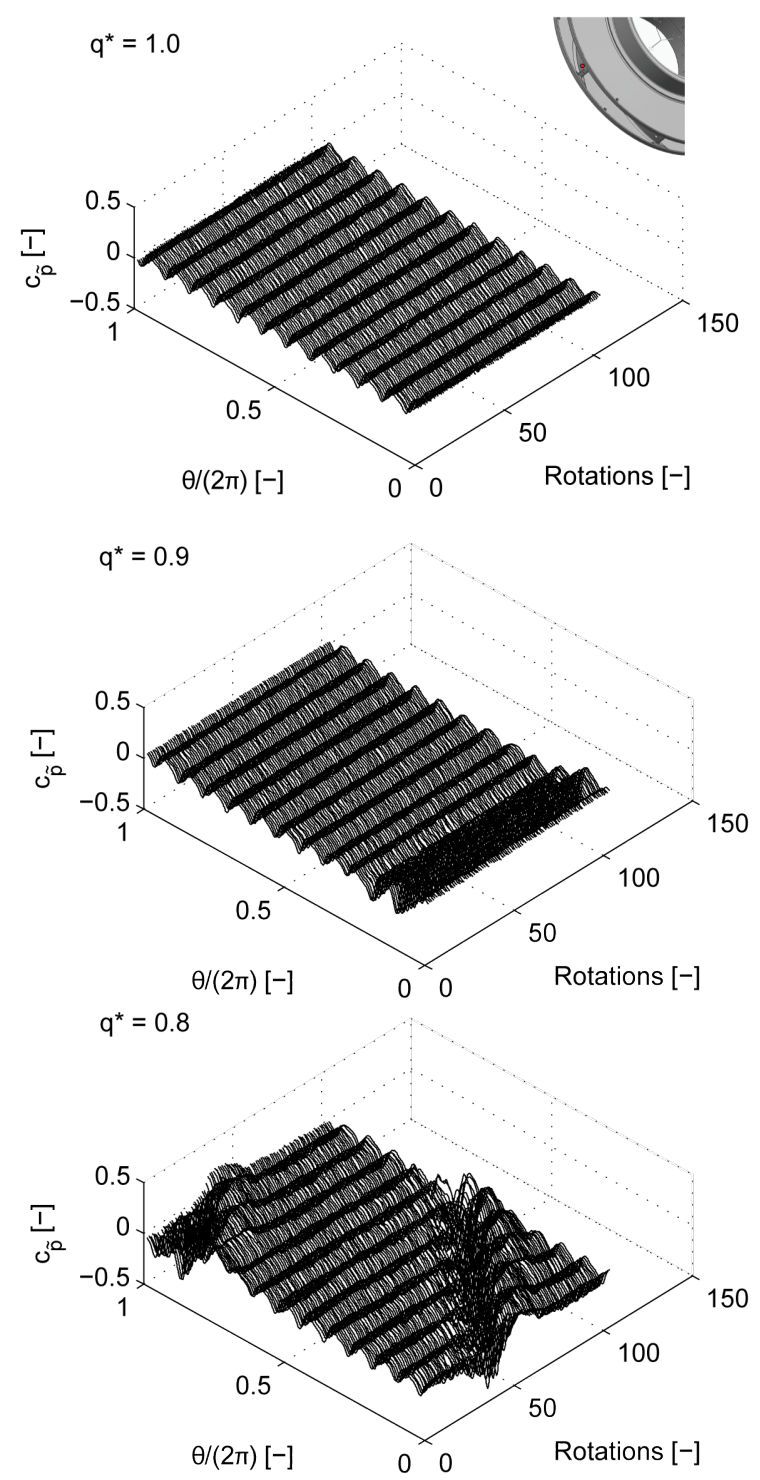

$q^{*}=0.6$

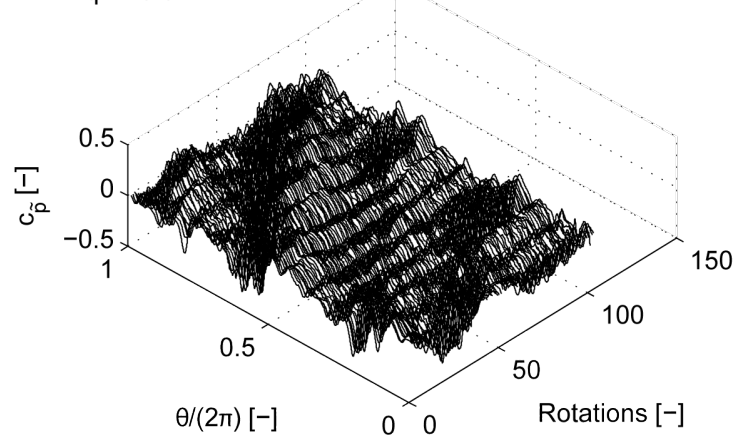

Figure 8. PRESSURE FLUCTUATIONS AT IMPELLER TRAILING EDGE FOR DIFFERENT FLOWRATES AT 1500 RPM 


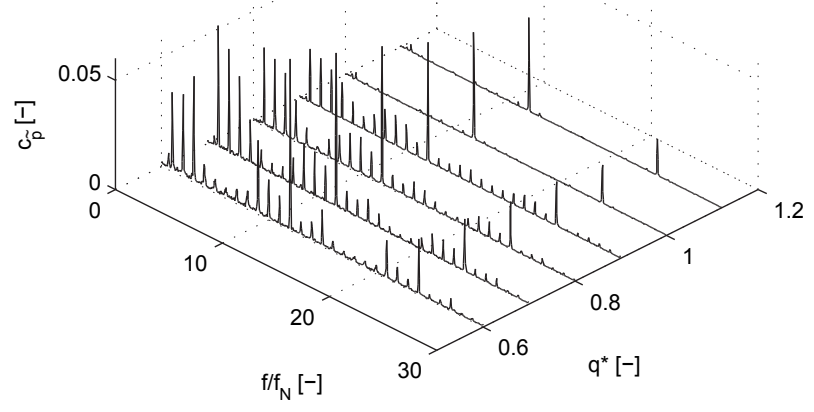

Figure 9. PRESSURE FLUCTUATION AMPLITUDES AT IMPELLER TRAILING EDGE FOR DIFFERENT FLOWRATES

\section{Stationary domain data}

Fig.10 depicts the pressure fluctuations in the vaned diffuser of the model pump at a position close to the rotor-stator interface at $\mathrm{q}^{*}=0.8$. At this flow rate, one stall cell is slowly propagating in the direction of the impeller rotation (see Fig.8). The waterfall plot of the dimensionless pressure fluctuations in the diffuser exhibited clearly the passage of the stall cell as a strong increase of the pressure during 20 impeller rotations. This increase repeats after 140 impeller rotations, when the stall cell finished one revolution. Fig.11 shows the phase averaged pressure fluctuations

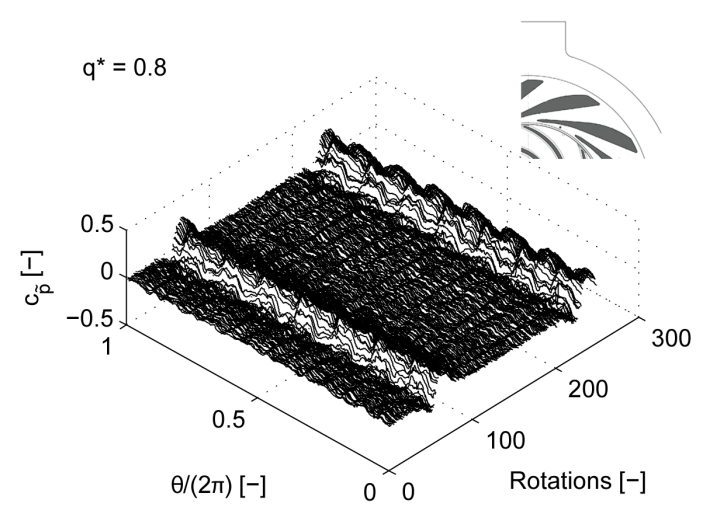

Figure 10. PRESSURE FLUCTUATIONS CLOSE TO ROTOR-STATOR INTERFACE

in one of the diffuser throats. This phase average has been calculated as the average of $c_{\tilde{p}}$ for each impeller revolution. Three different zones can be observed in the dimensionless pressure:
A Normal, undisturbed flow

B Pressure locally reduced due to the deviation of flow from the neighboring diffuser channel (in the direction opposite to the rotational direction), which increases the flow rate for the observed diffuser channel

C Pressure locally increased due to the stall of the diffuser channel (flow rate through this diffuser channel is nearly zero or even negative)

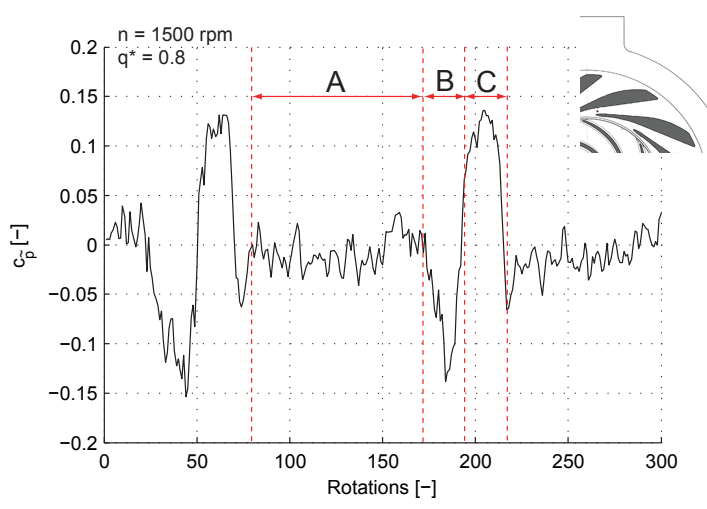

Figure 11. LOW FREQUENCY PRESSURE FLUCTUATIONS IN DIFFUSER THROAT

These perturbations in the pressure fluctuations extend into the rotor-stator interaction zone, affecting the frequency content of the pressure fluctuations experienced by the impeller (see Fig. 12 and Fig. 9) Fig.13 shows a waterfall plot of the pressure fluc-

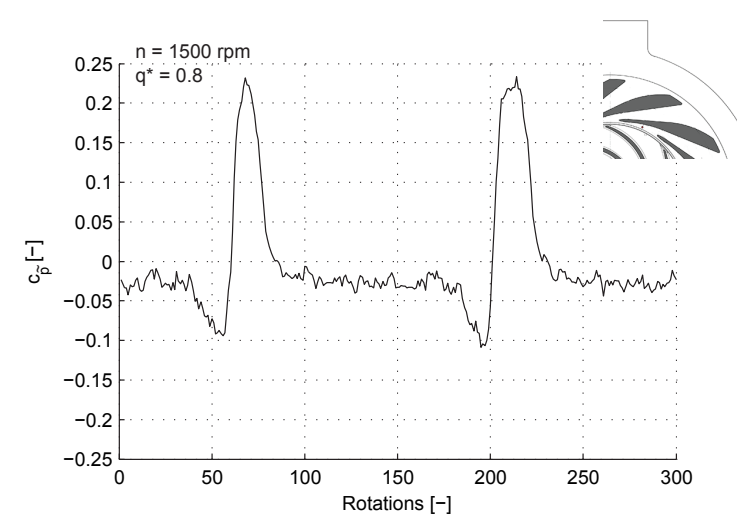

Figure 12. LOW FREQUENCY PRESSURE FLUCTUATIONS IN THE STATIONARY DOMAIN CLOSE TO ROTOR-STATOR INTERFACE

tuation amplitudes measured in the rotor stator interface. The 
spectra for each flow rate have been estimated as the average of 18 single spectra calculated using a Fast Fourier Transform. The resolution of these spectra is $1 \mathrm{~Hz}$, a Hanning window has been applied to each single spectrum. The pressure fluctuation spectra are dominated by the impeller blade passing frequency and its harmonics. When the rotating stall is present, pressure fluctuations at a very low frequency get visible, but the resolution of the spectra is not sufficient to use this information for further analysis. Neither the number of rotating stall cells nor their rotation

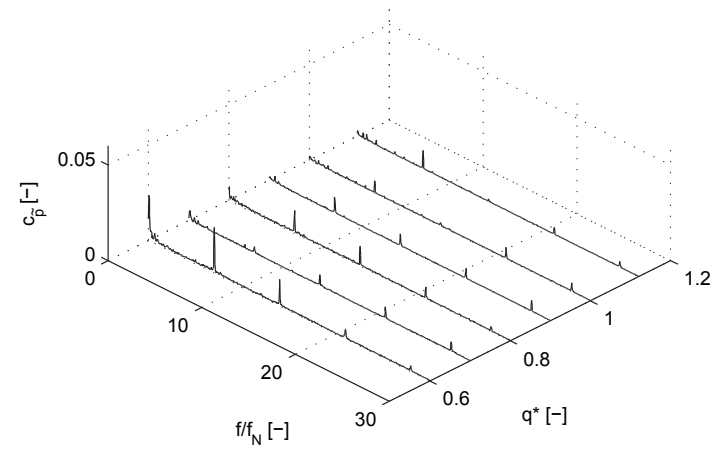

Figure 13. PRESSURE FLUCTUATION SPECTRA IN THE STATIONARY DOMAIN CLOSE TO ROTOR-STATOR INTERFACE

direction can be easily detected by the analysis of only one pressure sensor signal in the stationary domain. The identification of the number of stalled diffuser channels and their propagation direction can be achieved by the analysis of the pressure sensor array in the diffuser throat by means of cross-correlation based analysis or 2D-FFT. This is foreseen for future analysis.

\section{Influence of rotational speed}

The influence of the rotational speed of the pump on the pressure fluctuations has been investigated. Figure 14 presents the comparison of the phase averaged dimensionless pressure fluctuations measured close to the impeller trailing edge at 1500 $\mathrm{rpm}$ and $5000 \mathrm{rpm}$ at a relative flow rate of $\mathrm{q}^{*}=0.9$. The number of stalled diffuser channels has increased from one to two and its circumferential position has changed. The highest pressure peaks are found at two diffuser channels located diametrically opposed. The local maximum of the pressure is again followed by a local minimum of the pressure, indicating the deviation of the flow from the stalled diffuser channel to the adjacent channel in the rotational direction. Figure 15 presents the amplitude spectra of the dimensionless fluctuating pressure measured close to the impeller trailing edge shown in Fig.14. At 5000 RPM, the diffuser vane passing frequency remains dominant. Surprisingly, we observe a strong increase of the pressure fluctuation
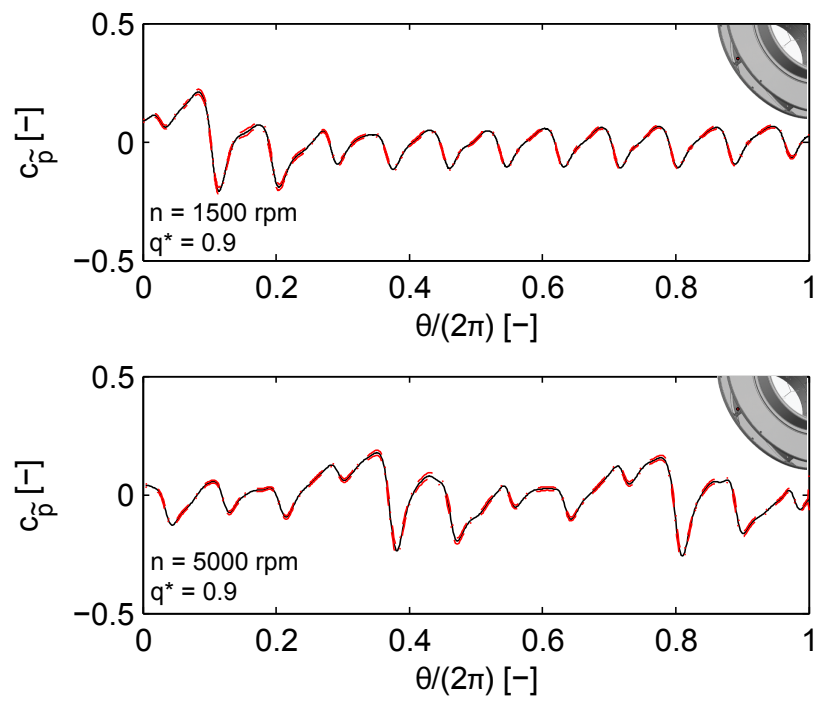

Figure 14. PHASE AVERAGED PRESSURE FLUCTUATIONS AT IMPELLER TRAILING EDGE FOR DIFFERENT ROTATIONAL SPEEDS
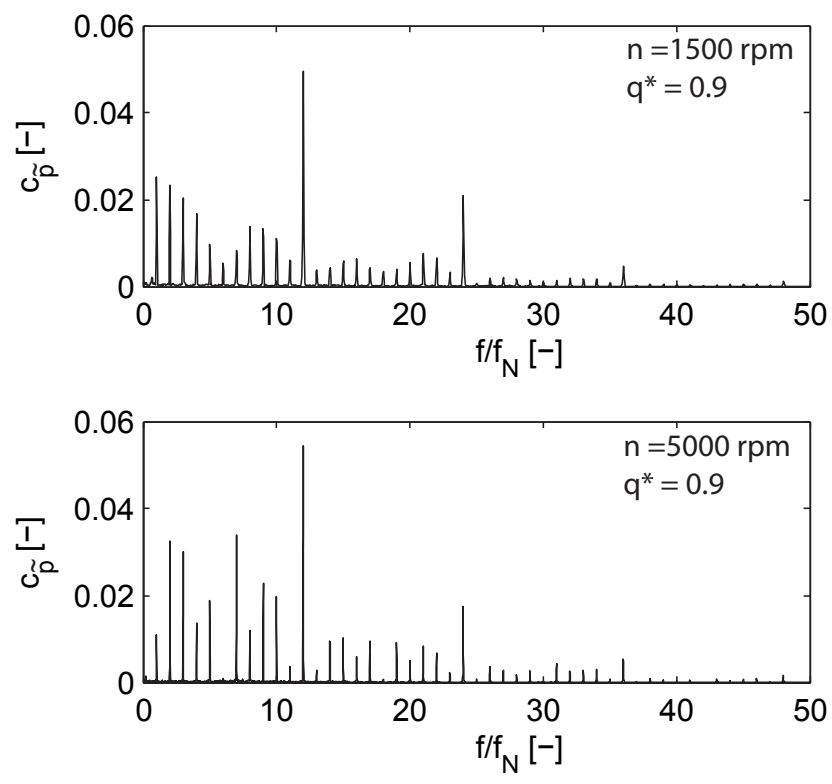

Figure 15. PHASE AVERAGED PRESSURE FLUCTUATIONS AT IMPELLER TRAILING EDGE FOR DIFFERENT ROTATIONAL SPEEDS

amplitude at $f=7 \cdot f_{N}$, the impeller blade passing frequency ! A possible explanation could be the occurrence of cavitation in the diffuser. In fact, similar observations have been made when cavitation occurs in Francis turbines as reported in [15]. With the presence of vapor cavities in the pump diffuser, the rotor stator interaction may be significantly stronger. This is why the resulting pressure pulses are well detected in the rotating frame leading 
to the rise of $f=7 \cdot f_{N}$ component.

A series of ramp tests at different relative flow rates has been performed, where the rotational speed has been lineary increased from 0 to $5500 \mathrm{rpm}$ within 20 seconds and the suction pressure was kept constant. Figure 16 shows a waterfall plot of the di-

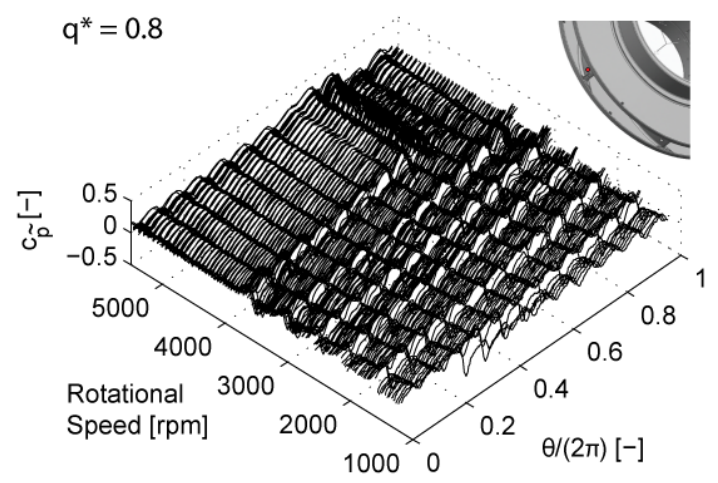

Figure 16. PART LOAD PRESSURE FLUCTUATIONS AT IMPELLER TRAILING EDGE FOR STEADILY INCREASING ROTATIONAL SPEED

mensionless fluctuating pressure measured close to the impeller trailing edge during a test at a relative flow rate of $q^{*}=0.8$. While at lower rotational speeds one rotating pressure peak can be observed, this peak stops its rotation at around $4200 \mathrm{rpm}$ and a stable stall establishes in two diffuser channels. The circumferential location of these channels is not the same as in the aforementioned test with a constant rotational speed at $5000 \mathrm{rpm}$. The measurements at different rotational speeds show the unsteady nature of the flow separation in the diffuser and the difficulties in the treatment of these phenomena in a systematic order. While the dependency of the rotation of the stall cells on the rotational speed of the pump indicates an influence of the Reynolds number on the propagation of the stall, it is not clear yet, to which extend other phenomena like cavitation, the interaction with the side room flow or the hydroacoustic properties of the casing may affect the development of a rotating stall.

\section{SUMMARY AND CONCLUSIONS}

In the present article, pressure fluctuation measurements in the impeller and the diffuser of a high speed centrifugal pump model have been presented. The pressure fluctuation measurements in the pump impeller have been performed with the help of a newly developed onboard data acquisition system, capable to acquire dynamic pressure fluctuation data in the rotating impeller with acquisition frequencies up to $100 \mathrm{kHz}$ at maximal rotational speeds of $6000 \mathrm{rpm}$. Flush mounted piezo-resistive miniature pressure transducers have been used in the stationary and rotating components of the pump. The performed pressure fluctuation measurements allowed the visualization of different pressure fluctuation patterns in the impeller and the diffuser at different flow rates. The measurements in the impeller trailing edge allowed the identification of stall in different diffuser channels, which were observed as rotating or stationary discontinuities in the periodic pressure fluctuation patterns. The number and the behavior of these discontinuities depend on the relative flow rate of the pump. When the stall was rotating, the propagation direction was the same as the rotation direction of the impeller. The rotational speed of the stall cells was less than $1 \%$ of the impeller rotational speed. The diffuser stall affected the spectral distribution of the pressure fluctuations experienced by the impeller. Sidebands with significant amplitudes appeared in the impeller pressure fluctuation spectra and consequently the impeller is not anymore mechanically excited only by contributions having the vane passing frequency and its harmonics but many more frequencies. This might have consequences on the mechanical behavior and the life span of the impeller, especially for high speed pumps and will be investigated further. Tests at variable rotational speeds unveiled a speed dependancy of the separation development, which can not be explained yet in a systematic order and which will be subject of further research.

\section{ACKNOWLEDGMENT}

The present study is supported by Sulzer Pumps, Swisselectric Research and the Swiss Competence Center of Energy and Mobility (CCEM). The authors would like to thank the staff of SULZER Pumps test field for their support in preparing and executing these experiments.

\section{REFERENCES}

[1] Frigne, P., and Van Den Braembussche, R., 1984. "Distinction between different types of impeller and diffuser rotating stall in a centrifugal compressor with vaneless diffuser.". J Eng Gas Turbines Power Trans ASME, 106, pp. 468-474.

[2] Haupt, U., Seidel, U., Abdel-Hamid, A., and Rautenberg, M., 1988. "Unsteady flow in a centrifugal compressor with different types of vaned diffusers". J Turbomach, 110(3), pp. 293-302.

[3] Filipenco, V., Deniz, S., Johnston, J., Greitzer, E., and Cumpsty, N., 2000. "Effects of inlet flow field conditions on the performance of centrifugal compressor diffusers: Part 1- discrete passage diffuser". Journal of Turbomachinery, 122(1), pp. 1-10.

[4] Deniz, S., Greitzer, E., and Cumpsty, N., 2000. "Effects of inlet flow field conditions on the performance of centrifugal compressor diffusers: Part 2-straight-channel diffuser". $J$. Turbomach., 122(1), pp. 11-21.

[5] Hergt, P., and Benner, R., 1968. "Visuelle Untersuchung 
der Strömung im Leitrad einer Kreiselpumpe". Schweizerische Bauzeitung, 86(40), pp. 716-720.

[6] Arndt, N., Acosta, A., Brennen, C., and Caughey, T., 1989. "Rotor-stator interaction in a diffuser pump". Journal of Turbomachinery, 111(3), pp. 213-221.

[7] Guo, S., and Maruta, Y., 2005. "Experimental investigations on pressure fluctuations and vibration of the impeller in a centrifugal pump with vaned diffusers". JSME International Journal, Series B: Fluids and Thermal Engineering, 48(1), February, pp. 136-143.

[8] Eisele, K., Zhang, Z., Casey, M., Gülich, J., and Schachenmann, A., 1997. "Flow analysis in a pump diffuser - part 1: Lda and ptv measurements of the unsteady flow". ASME Journal of Fluids Engineering, 119(4), pp. 968-977.

[9] Sano, T., Yoshida, Y., Tsujimoto, Y., Nakamura, Y., and Matsushima, T., 2002. "Numerical study of rotating stall in a pump vaned diffuser". Journal of Fluids Engineering, Transactions of the ASME, 124(2), pp. 363-370.

[10] Sinha, M., Pinarbasi, A., and Katz, J., 2001. "The flow structure during onset and developed states of rotating stall within a vaned diffuser of a centrifugal pump". Journal of Fluids Engineering, Transactions of the ASME, 123(3), pp. 490-499.

[11] Wang, H., and Tsukamoto, H., 2003. "Experimental and numerical study of unsteady flow in a diffuser pump at offdesign conditions". Journal of Fluids Engineering, Transactions of the ASME, 125(5), pp. 767-778.

[12] Farhat, M., Natal, S., Avellan, F., Paquet, F., Lowys, P. Y., and Couston, M., 2002. "Onboard Measurements of Pressure and Strain Fluctuations in a Model of low Head Francis Turbine. part 1 : Instrumentation”. In Proceedings of the 21st IAHR Symposium on Hydraulic Machinery and Systems, pp. 865-872.

[13] Avellan, F., Etter, S., Gummer, J. H., and Seidel, U., 2000. "Dynamic Pressure Measurements on a Model Turbine Runner and their Use in Preventing Runner Fatigue Failure". In Proceedings of the 20th IAHR Symposium, Charlotte, North Carolina, USA, August.

[14] Farhat, M., Avellan, F., and Seidel, U., 2002. "Pressure Fluctuation Measurements in Hydro Turbine Models". In Proceedings of the 9th International Symposium on Transport Phenomena and Dynamics of Rotating Machinery, Honolulu, Hawaii, USA, February.

[15] Farhat, M., Bourdon, P., Lavigne, P., and Simoneau, R., 1997. "Hydrodynamic aggressiveness of cavitating flows in hydro turbines". In Proceedings of the 1997 ASME Fluids Engineering Division Summer Meeting, FEDSM'97. Part 16 (of 24), Anon, ed., Vol. 4, ASME, pp. -. 